\title{
A 20-year multi-follow-up of hallucinations in schizophrenia, other psychotic, and mood disorders
}

\author{
V. M. Goghari ${ }^{1 *}$, M. Harrow ${ }^{2}$, L. S. Grossman ${ }^{2}$ and C. Rosen $^{2}$ \\ ${ }^{1}$ Departments of Psychology and Psychiatry, Hotchkiss Brain Institute, University of Calgary, Calgary, AB, Canada \\ ${ }^{2}$ Department of Psychiatry, University of Illinois-Chicago, Chicago, IL, USA
}

Background. Hallucinations are a major aspect of psychosis and a diagnostic feature of both psychotic and mood disorders. However, the field lacks information regarding the long-term course of hallucinations in these disorders. Our goals were to determine the percentage of patients with hallucinations and the relationship between hallucinations and recovery, and work attainment.

Method. The present study was a prospective evaluation of the 20-year trajectory of hallucinations in 150 young patients: 51 schizophrenia, 25 schizoaffective, 25 bipolar with psychosis, and 49 unipolar depression. The patients were studied at an index phase of hospitalization for hallucinations, and then reassessed longitudinally at six subsequent follow-ups over 20 years.

Results. The longitudinal course of hallucinations clearly differentiated between schizophrenia and bipolar disorder with psychosis, and suggested some diagnostic similarities between schizophrenia and schizoaffective disorder, and between bipolar disorder and schizoaffective disorder and depression. Frequent or persistent hallucinatory activity over the 20-year period was a feature of $40-45 \%$ of schizophrenia patients. The early presence of hallucinations predicted the lack of future periods of recovery in all patients. Increased hallucinatory activity was associated with reduced work attainment in all patients.

Conclusions. This study provides data on the prospective longitudinal course of hallucinations, which were previously unavailable to the field, and are one of the key features of psychosis in major psychiatric disorders. This information on the clinical course of major psychiatric disorders can inform accurate classification and diagnosis.

Received 6 June 2012; Revised 17 August 2012; Accepted 20 August 2012 ; First published online 4 October 2012

Key words: Bipolar disorder, depression, psychosis, schizoaffective, schizophrenia.

\section{Introduction}

Understanding the longitudinal course of symptoms is considered essential to understanding the disorder (Kraepelin, 1907; Bleuler, 1950). Longitudinal studies have contributed vastly to our knowledge of schizophrenia, demonstrating that the disorder is largely chronic and that schizophrenia patients tend to have worse outcomes than patients with other major psychiatric disorders, including schizoaffective disorder, bipolar disorder and unipolar affective disorders (for a review, see McGlashan, 1988). Furthermore, longitudinal studies have demonstrated that the majority of the loss of functioning in schizophrenia takes place in the first 5-10 years, and then the disorder remains relatively stable (McGlashan, 1988). Last, longitudinal studies have demonstrated that outcome is

* Address for correspondence: V. M. Goghari, Ph.D., R. Psych., Department of Psychology, University of Calgary, Administration Building, 2500 University Drive NW, Calgary, AB, Canada T2N 1N4. (Email: vina.m.goghari@ucalgary.ca) heterogeneous in schizophrenia (McGlashan, 1988). However, knowledge regarding the longitudinal course and predictive value of certain symptoms, such as hallucinations, in common psychiatric disorders is relatively absent (Waters et al. 2012).

Hallucinations are a central diagnostic feature of psychotic disorders and can also occur in mood disorders. These symptoms are also some of the most noticeable signs of a psychiatric disorder and are often the reason why individuals are referred to the hospital for diagnostic assessment and treatment. Previous research has suggested that positive symptoms (Pfohl \& Winokur, 1982; Mancevski et al. 2007) and hallucinations specifically (Gur et al. 1996) tend to decrease as patients age in schizophrenia; however, prospective longitudinal demonstration of how frequently different groups of psychotic and mood disorder patients experience hallucinations and whether the longitudinal trajectory of hallucinations can differentiate between these diagnostic categories is lacking.

Given the importance of predicting real-world dayto-day functioning in patients, further information on 
the relationship between hallucinations and independent living skills, especially work attainment, is necessary. For example, in Canada in 2004, the total direct healthcare and non-healthcare cost for schizophrenia was estimated at $\$ 2$ billion, whereas the productivity loss was estimated at $\$ 5$ billion (Goeree et al. 2005). Understanding the determinants of work disability in individuals with psychotic disorders could lead to a substantial economic benefit. Prominent reviews have suggested that work attainment is predicted by cognition, and, to a lesser degree, by negative symptoms, with positive symptoms playing a minimal role (Green et al. 2000). However, other studies have provided support that positive symptoms, such as delusions, are associated with work functioning (Harrow et al. 2004; Harrow \& Jobe, 2010). The current study assessed the longitudinal trajectory of hallucinations and its relation to real-world functioning.

In summary, the goals of this investigation were to provide prospectively collected information to the field regarding: (1) the longitudinal course of hallucinations in patients with psychotic and mood disorders; (2) how the course of hallucinations differs in these disorders; and (3) whether hallucinations are associated with real-world functioning and in particular with work attainment.

\section{Method}

\section{Participants and measures}

The present study is based on data from the Chicago Follow-up Study, a prospectively designed, longitudinal, multi-follow-up research program studying psychopathology and recovery in psychiatric disorders (Harrow et al. 2004; Harrow \& Jobe, 2005). Institutional Review Board approval was obtained from the University of Illinois-Chicago and signed informed consent was obtained from all participants. The sample for this investigation consisted of 150 patients, who were studied prospectively at an index phase of hospitalization and at six follow-up periods over 20 years. Using the Research Diagnostic Criteria (Spitzer et al. 1978), four major patient groups were studied-51 schizophrenia, 25 schizoaffective (23 schizoaffective-depressed and three schizoaffective-manic), 25 bipolar with psychosis, and 49 non-psychotic unipolar depression patients - based on research diagnoses at the index hospitalization. The unipolar depression patients were included as a nonpsychotic psychiatric group for comparison at the index hospitalization, and as a comparison with bipolar patients over the 20-year follow-up period. The research diagnoses were based on at least one of two structured interviews: (1) the Schedule for Affective Disorders and Schizophrenia (SADS; Endicott \& Spitzer, 1978); and/or (2) the Schizophrenic State Inventory (Grinker \& Harrow, 1987). Satisfactory inter-rater reliability was established for diagnosis (e.g. a $\kappa$ of 0.88 for schizophrenia).

The patients were then reassessed, individually, at six subsequent follow-ups over a 20-year period. The follow-ups occurred at approximately 2, 4.5, 7.5, 10, 15 and 20 years after the index hospitalization. Information for the 20-year follow-up was available for $73 \%$ of the original sample assessed. There were no notable differences in attrition for the different diagnostic groups. The sample assessed at the 20-year follow-up did not differ significantly on major demographic variables from the subsamples that were assessed at the index hospitalization and 2-year follow-up, but not assessed at the 20-year follow-up.

The follow-up evaluations involved structured interviews on symptoms and functioning. These interviews were conducted by trained raters who were blind to the diagnosis. Hallucinations at the index hospitalization and at each follow-up were assessed with the SADS for the past month (Endicott \& Spitzer, 1978). Hallucinations were rated on a three-point scale as follows: $1=$ hallucination absent; $2=$ weak or equivocal hallucination; $3=$ full hallucination present (Marengo et al. 2000; Ventura et al. 2011). This included auditory hallucinations, such as audible thoughts, voices commenting, voices conversing, voices arguing; visual hallucinations; olfactory hallucinations; and somatic or tactile hallucinations. This paper combined across the different types of hallucinations to obtain a binary value coding the presence or absence of any hallucination. The Global Assessment Scale (GAS) was also completed at every follow-up, rated for the past year, as a global measure of functioning (Endicott et al. 1976).

\section{Operational criteria for recovery}

Social and work attainment measures were acquired at every follow-up. The criteria for period of 'recovery' during a follow-up year were defined operationally as the absence of positive or negative symptoms and no re-hospitalizations during the follow-up year. Recovery also required partially adequate (or better) work and social functioning [scores of ' 2 - employed half-time or greater' on the Strauss-Carpenter scales (Strauss \& Carpenter, 1972)]. To avoid confounding the variables when assessing the relationship between hallucinations and recovery, we assessed whether index hallucinations predicted recovery over the next 20 years and also whether hallucinations at the 2-year follow-up predicted recovery over the next 18 years. 
Table 1. Participant characteristics

\begin{tabular}{|c|c|c|c|c|}
\hline & Schizophrenia & Schizoaffective & Bipolar with psychosis & Depression \\
\hline Subjects, $n$ & 51 & 25 & 25 & 49 \\
\hline \multicolumn{5}{|l|}{ Mean age, years (S.D.) } \\
\hline At index hospitalization & $23.1(3.5)$ & $22.4(3.4)$ & $24.5(6.4)$ & $23.2(3.4)$ \\
\hline \multicolumn{5}{|l|}{ At follow-up } \\
\hline 2-year follow-up & $25.6(3.6)$ & $24.7(3.6)$ & $27.6(5.7)$ & $25.3(3.3)$ \\
\hline 4.5-year follow-up & $28.4(3.9)$ & $27.4(3.4)$ & $30.0(5.4)$ & $27.7(3.6)$ \\
\hline 7.5-year follow-up & $30.8(3.7)$ & $29.7(3.8)$ & $31.7(5.8)$ & $30.4(3.7)$ \\
\hline 10-year follow-up & $34.0(4.2)$ & $33.0(3.9)$ & $34.5(4.4)$ & $33.8(3.7)$ \\
\hline 15-year follow-up & $38.3(4.1)$ & $37.0(3.4)$ & $40.1(6.4)$ & $37.9(4.4)$ \\
\hline 20-year follow-up & $43.4(3.9)$ & $42.4(4.2)$ & $45.3(6.0)$ & $43.2(3.8)$ \\
\hline Sex, \% male & 67 & 56 & 48 & 41 \\
\hline \multicolumn{5}{|l|}{ Ethnicity, \% } \\
\hline White & 63 & 88 & 64 & 76 \\
\hline Black & 37 & 12 & 32 & 22 \\
\hline Other & 0 & 0 & 4 & 2 \\
\hline Education, years completed (s.D.) & $12.7(2.0)$ & $13.6(1.95)$ & $13.5(2.5)$ & $14.4(2.1)$ \\
\hline \multicolumn{5}{|l|}{ Prior hospitalizations } \\
\hline Mean number (S.D.) & $1.7(2.0)$ & $1.2(1.6)$ & $2.4(2.8)$ & $0.3(0.5)$ \\
\hline Range & $0-9$ & $0-6$ & $0-12$ & $0-2$ \\
\hline
\end{tabular}

S.D., Standard deviation.

Hence, that period of hallucinations that was used as a predictor was not included in the computation of recovery over the subsequent follow-ups.

\section{Statistical analyses}

All available data were used in analyses. Unipolar depression patients were excluded from correlation and regression analyses that assessed hallucinations at the index hospitalization. The generalized estimation equation in SPSS (SPSS, Inc., USA) was used to analyse the binary logistic repeated-measures hallucinatory data. The model and pair-wise least-squares difference post hoc tests were used to analyse the effect of psychiatric group and follow-up period on hallucinatory activity. Pearson's $\chi^{2}$ tests were used to compare groups at the index hospitalization and recovery status. Linear-by-linear association $\chi^{2}$ tests were used to compare groups for chronicity (none, some, frequent, chronic) as they account for increasing chronicity. Last, we assessed whether hallucinations predicted work attainment and recovery using logistic regressions. For all analyses, hallucination data were coded as being present (score of 2 or 3 on the threepoint scale described above) or absent (score of 1 on the three-point scale described above).

In this study, we included patients who attended five or six follow-up assessments (out of a possible six). Diagnostic groups did not differ in number of follow-ups attended $\left[\chi^{2}(3)=1.80, p=0.61\right]$. Nor was the presence of hallucinations at the index hospitalization associated with the number of follow-ups attended $\left[\chi^{2}(3)=0.47, p=0.49\right]$.

Additionally, we investigated the effects of sex by adding it as an additional factor in the generalized estimation equation model. There were no significant effects of sex, sex $\times$ follow-up period interaction, or sex $\times$ group interaction $\left(\chi^{2}<2.29, p^{\prime} s>0.81\right)$. Therefore, analyses were collapsed across sex.

\section{Results \\ Participant characteristics}

Table 1 describes participant characteristics. There were no significant differences between groups for age $\left(F_{3,146}=1.41, p=0.24\right)$ for the index hospitalization; however, there were trends for differences at the 2-year and 4.5-year follow-ups in the overall analyses of variance $\left(F^{\prime} s=2.28-2.51, p^{\prime} s=0.06-0.08\right)$, demonstrating that bipolar patients were older than all other patients at the 2-year follow-up ( $\left.p^{\prime} s=0.01-0.04\right)$ and older than schizoaffective and depression patients at the 4.5-year follow-up ( $\left.p^{\prime} \mathrm{s}=0.02\right)$. There was a trend for a sex difference between groups $\left[\chi^{2}(3)=7.09\right.$, $p=0.07]$, with the depression group having more female participants than the schizophrenia group $\left[\chi^{2}(1)=6.72, p=0.01\right]$. Groups did not differ for ethnicity $\left[\chi^{2}(6)=8.95, p=0.18\right]$. Groups differed on educational achievement $\left(F_{3,146}=5.11, p=0.002\right)$, with 


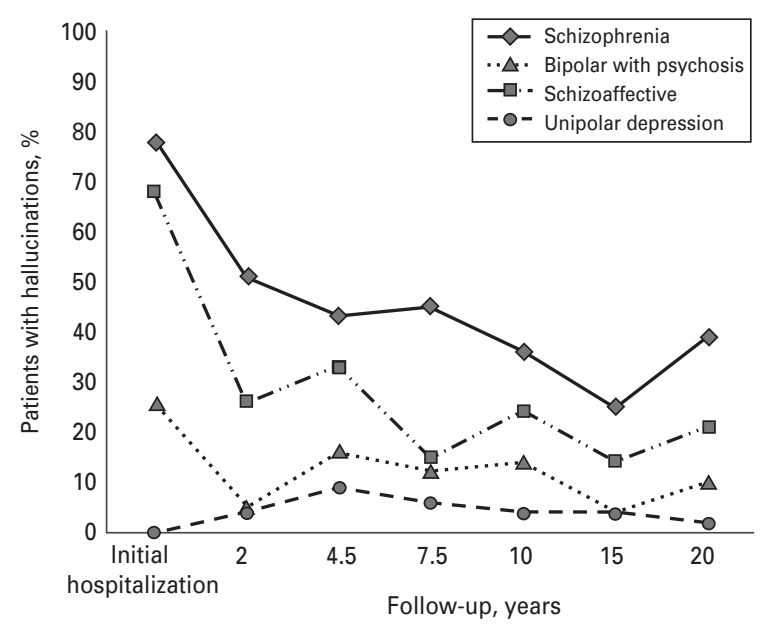

Fig. 1. Longitudinal trajectory of hallucinations in patients with schizophrenia, schizoaffective disorder, bipolar disorder with psychosis and unipolar depression over a 20-year period.

schizophrenia patients having a lower level of education than depression patients $(p<0.001)$. Higher education level was associated with less hallucinatory activity at the index hospitalization and at all the follow-ups $\left(r=-0.20\right.$ to $-0.30, p^{\prime}$ s $\left.<0.0001-0.05\right)$. Greater index education level was also associated with a greater chance of having a period of recovery over the next 20 years $(r=0.22, p=0.006)$. There were no differences in social class between groups $\left[\chi^{2}(12)=15.54, p=0.21\right]$. Groups differed for number of previous hospitalizations $\left(F_{3,146}=8.81, p<0.001\right)$. Schizophrenia $(p<0.001)$, schizoaffective $(p=0.04)$ and bipolar with psychosis $(p<0.001)$ patients had a greater number of previous hospitalizations compared with depression patients. Additionally, bipolar patients had a greater number of previous hospitalizations compared with schizoaffective patients $(p=0.02)$. Overall, $47 \%$ of patients were firstadmission patients and $73 \%$ had one or no previous hospitalizations prior to the index hospitalization. A greater number of hospitalizations as measured at the index hospitalization was not associated with a greater level of hallucinations at the index hospitalization $(r=-0.06, p=0.59)$. However, a greater number of hospitalizations at index was associated with being less likely to have a period of recovery over the next 20 years $(r=-19, p=0.02)$. Last, groups differed at all six follow-ups for global assessment of functioning (GAS) ( $\left.F^{\prime} \mathrm{s}=5.09-13.79, \quad p^{\prime} \mathrm{s}<0.001-0.003\right)$. Schizophrenia patients did not differ from schizoaffective patients at any of the follow-ups ( $p^{\prime}$ s $\left.>0.09\right)$, but did have poorer functioning than bipolar with psychosis patients at the 4.5-year, 7.5-year, 10-year and 20-year follow-ups ( $\left.p^{\prime} s<0.02\right)$ and depression patients at all follow-ups $\left(p^{\prime} s<0.001\right)$. Schizoaffective patients had poorer functioning than bipolar with psychosis patients at the 4.5-year follow-up $(p=0.009)$ and depression patients at the 2-year, 4.5-year, 7.5-year and 10-year follow-ups ( $p$ 's $<0.009)$. Last, bipolar with psychosis patients had poorer functioning than the depression patients at the 7.5-year and 15-year follow-ups ( $\left.p^{\prime} s<0.01\right)$.

\section{Longitudinal trajectory of hallucinations in psychotic and mood disorders over a 20-year period}

There was a significant effect of group on hallucination status at the index hospitalization $\left[\chi^{2}(3)=69.48\right.$, $p<0.001$; see Fig. 1]. Schizophrenia patients did not differ from schizoaffective patients $\left[\chi^{2}(1)=0.79\right.$, $p=0.37]$ at the index hospitalization. Both schizophrenia $\left[\chi^{2}(1)=17.44, p<0.001\right]$ and schizoaffective patients $\left[\chi^{2}(1)=8.43, p=0.004\right]$ had more hallucinatory activity compared with bipolar with psychosis patients. Schizophrenia, schizoaffective and bipolar with psychosis patients had more hallucinations at the index hospitalization compared with non-psychotic unipolar depression patients $\left(\chi^{2}=13.68-61.20, p^{\prime}\right.$ s $<0.001)$, who were used as a psychiatric control group.

A generalized estimating equation model was used to evaluate the difference between the index hospitalization and follow-up periods for the three diagnostic groups (schizophrenia, schizoaffective and bipolar with psychosis disorders) at the index hospitalization. All three groups had more hallucinations at the index hospitalization compared with each follow-up period $\left(\chi^{2} s=14.05-29.98, p^{\prime} s<0.001\right)$. There was no interaction between time and group for any of the comparisons ( $p^{\prime}$ s >0.17).

A second generalized estimating equation model evaluated for differences in hallucinatory status between the four patient groups after the index phase over the six follow-up periods. The model demonstrated a significant effect of group $\left[\chi^{2}(3)=26.78\right.$, $p<0.001]$. Least-squares difference pair-wise comparisons demonstrated that schizophrenia patients, collapsing across all follow-up periods, had more hallucinations than schizoaffective patients $(p=0.03)$, bipolar with psychosis patients $(p<0.001)$ and depression patients $(p<0.001)$. Schizoaffective patients did not differ significantly from bipolar with psychosis patients $(p=0.096)$, but had more hallucinatory activity than depression patients $(p=0.01)$. Bipolar with psychosis patients did not differ from depression patients $(p=0.37)$.

There was also a significant main effect of followup period $\left[\chi^{2}(5)=13.88, p=0.02\right]$. There was more hallucinatory activity during the 4.5-year $(p<0.001)$ and 7.5-year $(p=0.005)$ follow-up periods than during 


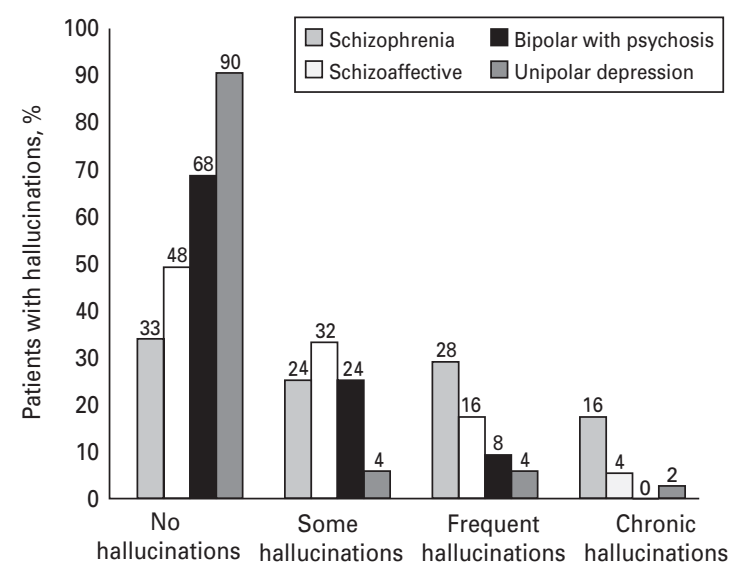

Fig. 2. Chronicity of hallucinations in patients with schizophrenia, schizoaffective disorder, bipolar disorder with psychosis and unipolar depression over a 20-year period.

the 15-year follow-up. During the 4.5-year follow-up, there was also more hallucinatory activity than at the 20-year follow-up period ( $p=0.02)$.

Additionally, the generalized estimating equation model demonstrated a trend towards a group $\times$ follow-up period interaction $\left[\chi^{2}(15)=23.72, p=0.07\right]$. Schizophrenia patients had more hallucinations compared with schizoaffective patients at the 2-year and 7.5-year follow-ups ( $p^{\prime} s<0.03$ ). Schizophrenia patients had more hallucinations at all follow-ups over the 20 years compared with bipolar with psychosis patients ( $p^{\prime}$ s $<0.04$ ) and depression patients ( $p$ 's $<0.004)$. Schizoaffective patients had more hallucinations than bipolar with psychosis patients at the 2-year follow-up $(p=0.04)$, and depression patients at the 2-year, 4.5-year, 10-year and 20-year follow-ups $(p$ 's $<0.03)$. Bipolar with psychosis patients did not differ from depression patients at any of the followups $\left(p^{\prime} \mathrm{s}>0.22\right)$.

\section{Chronicity of hallucinations in psychotic and mood disorders over a 20-year period}

Fig. 2 depicts the percentage of patients in the four psychiatric groups who had chronic hallucinations (i.e. hallucinations at all follow-up periods), frequently recurring hallucinations (i.e. hallucinations at three or more of the follow-up periods), some hallucinations, and no hallucinations. A linear-by-linear association $\chi^{2}$ test taking into account order effects found that there was a significant difference in the chronicity of hallucinations between groups $\left[\chi^{2}(1)=33.15\right.$, $p<0.001$ ]. Follow-up linear-by-linear association $\chi^{2}$ tests revealed that schizophrenia patients demonstrated greater chronicity of hallucinations compared with schizoaffective patients $\left[\chi^{2}(1)=3.74, p=0.05\right]$, bipolar with psychosis patients $\left[\chi^{2}(1)=11.22, p=0.001\right]$ and depression patients $\left[\chi^{2}(1)=26.86, p<0.001\right]$. Schizoaffective patients did not differ from bipolar with psychosis patients in chronicity $\left[\chi^{2}(1)=2.63\right.$, $p=0.11]$, but did show greater chronicity than depression patients $\left[\chi^{2}(1)=9.69, p=0.002\right]$. Last, bipolar with psychosis patients did not have more chronic hallucinatory activity than depression patients $\left[\chi^{2}(1)=2.01, p=0.16\right]$.

\section{Relationship between hallucinations and recovery}

We conducted $\chi^{2}$ tests to investigate whether the presence of hallucinations at the index hospitalization and 2-year follow-up were associated with having a period of recovery over the next 18 or 20 years. Patients who did not have hallucinations at the index hospitalization were not more likely to have at least one period of global recovery over the next 20 years $\left[\left(\chi^{2}(1)=1.29, p=0.26\right]\right.$. However, not having hallucinations at the 2-year follow-up was associated with having at least one period of global recovery over the next 18 years $\left[\chi^{2}(1)=27.57, p<0.001\right]$. For those patients who had a period of recovery, $90 \%$ had no hallucinations at the 2-year follow-up.

We also analysed the pattern specifically in schizophrenia patients, as recovery in schizophrenia has been a source of theoretical discussion. Schizophrenia patients who did not have hallucinations at the index hospitalization were significantly more likely to have at least one global recovery period over the next 20 years $\left[\chi^{2}(1)=3.79, p=0.05\right]$. The same relationship was present for the 2-year follow-up, predicting at least one global recovery period over the next 18 years $\left[\chi^{2}(1)=6.33, p=0.01\right]$.

\section{Relationship between hallucinations and work attainment}

We conducted six $\chi^{2}$ tests to investigate whether the presence of hallucinations at the six follow-up periods predicted the ability to work more than part time (see Fig. 3). Collapsing across all diagnostic groups, the presence of hallucinations predicted working less than part time, whereas the absence of hallucinations predicted working more than part time at all six follow-ups $\left(\chi^{2 \prime} s=5.83-32.60, p^{\prime} s\right.$ $<0.001-0.02$ )

We also analysed the pattern specifically in schizophrenia patients. Similar to when all patients were analysed, a lack of hallucinations was associated with working more than part time at all of the follow-ups in schizophrenia, which reached significance at the 4.5-, $7.5-, 10-, 15-$ and 20-year follow-ups $\left(\chi^{2 \prime} \mathrm{s}=3.67-15.11\right.$, $p^{\prime}$ s $\left.<0.001-0.055\right)$. 
No hallucinations $\square$ Hallucinations

(a)

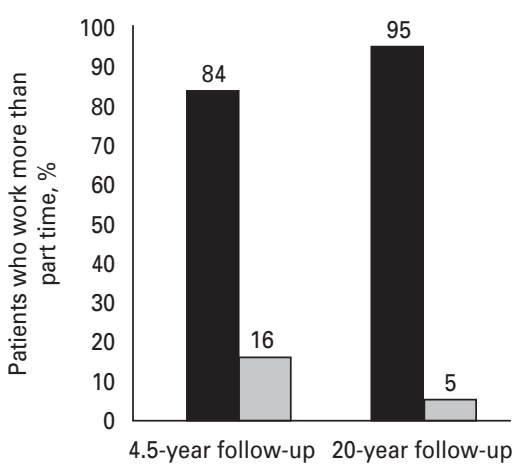

(b)

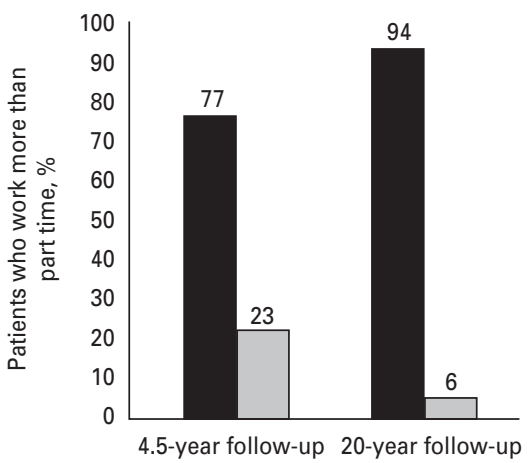

Fig. 3. Association of hallucination status with work attainment in all patients $(a)$ and schizophrenia patients $(b)$ specifically at the 4.5-year and 20-year follow-ups.

\section{Do hallucinations predict future recovery and work attainment beyond global functioning and delusions in all patients?}

The presence of hallucinations at the 2-year followup in all patients predicted a future period of recovery over the next 18 years $\left[\left(\chi^{2}(1)=5.94, p=0.02\right]\right.$, above and beyond the relationship between recovery and global functioning $\left[\chi^{2}(1)=4.29, p=0.04\right]$. Moreover, hallucinations at the 2-year follow-up predicted a future period of recovery over the next 18 years $\left[\chi^{2}(1)=12.15, p<0.001\right]$, above and beyond the relationship between recovery and delusions $\left[\chi^{2}(1)=\right.$ 1.998, $p=0.16]$.

Additionally, in all patients, we found that hallucinations were associated with work attainment $\left(\chi^{2 \prime} s=4.62-5.34, p^{\prime} s=0.02-0.03\right)$, above and beyond the relationship between work attainment and global functioning for the 7.5-year and 20-year follow-ups $\left(\chi^{2 \prime} s=15.74-23.53, p^{\prime} s<0.001\right)$. Furthermore, hallucinations were associated with work attainment at the 2-year, 4.5-year, 7.5-year and 20-year follow-ups $\left(\chi^{2}\right.$ s $=3.77-13.27, p^{\prime}$ s $\left.<0.001-0.05\right)$, above and beyond the relationship between work attainment and delusions $\left(\chi^{2} \mathrm{~s}=0.09-11.23, p^{\prime} \mathrm{s}<0.001-0.76\right)$.

\section{Antipsychotic medications}

At the 2-year follow-up, 64\% of schizophrenia patients, $58 \%$ of schizoaffective patients, $19 \%$ of bipolar with psychosis patients and $11 \%$ of unipolar depression patients were on antipsychotic medications. The figures remained relatively consistent over the next 18 years. At the 20-year follow-up, 55\% of schizophrenia patients, $63 \%$ of schizoaffective patients, $27 \%$ of bipolar with psychosis patients and
$10 \%$ of depression patients were on antipsychotic medications.

The relationship between antipsychotic medication status and hallucinations was assessed in schizophrenia and schizoaffective patients given that there were sufficient numbers for analyses. Schizophrenia patients not on any antipsychotic medication tended to have less hallucinatory activity than schizophrenia patients on antipsychotic medications, which reached significance at the 2-year, 4.5-year, 7.5-year, 10-year and 20-year follow-ups $\left(\chi^{2 \prime} \mathrm{s}=4.33-5.74, p^{\prime} \mathrm{s}=0.02\right.$ $0.04)$. Additionally, schizoaffective patients not on any antipsychotic medications had less hallucinatory activity at the 10-year follow-up than schizoaffective patients on antipsychotic medications $\left[\chi^{2}(1)=5.26\right.$, $p=0.02]$.

\section{Discussion}

Our understanding and categorization of severe mental disorders are based on our assumptions about longitudinal course. Despite the importance of hallucinations as a major type of psychotic symptom, systematic data on how many psychotic and mood disorder patients have frequent or persistent hallucinatory activity on a longitudinal basis have not previously been available to the field. The goals of this investigation were to determine: (1) the longitudinal course of hallucinations in patients with psychotic and mood disorders; (2) whether the course of hallucinations differs in these disorders; and (3) whether hallucinations can predict real-world functioning. This study demonstrated that the longitudinal course of hallucinations was useful in differentiating between disorders, and valuable in predicting the future clinical course of severe mental disorders. 


\section{Longitudinal trajectory of hallucinations in psychotic and mood disorders over a 20-year period}

Schizophrenia, schizoaffective and bipolar with psychosis patients all had significantly more hallucinations at the index hospitalization than at the follow-ups. Although in this study having more hallucinations in the early years was confounded by selection of patients from hospitals at index, this finding is consistent with other studies that suggest that the early period is typically the worst symptomatic period in the course of psychosis (Harding et al. 1987; McGlashan, 1988; Arndt et al. 1995). These data are consistent with previous research that demonstrates that positive symptoms tend to decrease over the lifespan of schizophrenia patients (Pfohl \& Winokur, 1982; Gur et al. 1996; Schultz et al. 1997; Mancevski et al. 2007). However, these data also demonstrate that there were a substantial number of patients who continued to show hallucinations after their index hospitalization. Of the patients, $54 \%$ of schizophrenia patients and $20 \%$ of schizoaffective patients showed frequent or chronic hallucinations over the 20-year course of the study. Comparatively, bipolar with psychosis and depression patients were less likely to have frequent or chronic hallucinations $(8 \%$ and $6 \%$, respectively). Overall, these data fit with long-held views that schizophrenia patients are more vulnerable to chronic courses of psychotic symptomatology compared with other disorders with psychosis (Kraepelin, 1907 ; Bleuler, 1950 ; McGlashan, 1988).

\section{Hallucinatory status differentiates between groups over the course of 20 years}

One of the main issues examined in this study was whether hallucinatory status over the course of 20 years is useful in differentiating between major mental disorders. Given that differential diagnosis between psychotic and mood disorders is often difficult, further knowledge regarding how symptoms differ in disorders is of key interest to the field. The data indicate that although schizophrenia and schizoaffective patients had similar levels of hallucinations at the index hospitalization, the longitudinal course of hallucinations differentiated between the two disorders. Schizophrenia patients had more hallucinations than schizoaffective patients collapsed across all follow-up periods, specifically at the earlier followups ( 2 and 7.5 years), and more chronic hallucinations. Additionally, the longitudinal course of hallucinations differentiated schizophrenia patients from bipolar with psychosis and unipolar depression patients over the 20 years. At the index hospitalization and throughout the 20-year follow-up period, schizophrenia patients had more hallucinations than both groups. Consistent with previous research, this provides further evidence of the greater vulnerability of schizophrenia patients to psychotic symptoms (McGlashan, 1988).

Another important diagnostic comparison is between mood disorders with psychosis and schizoaffective patients. This study found that schizoaffective patients had a more nuanced pattern of hallucinatory status compared with bipolar with psychosis and depression patients. Schizoaffective patients had more hallucinations at the index hospitalization and in the 2-year follow-up period compared with bipolar with psychosis patients. This suggests that the early course of hallucinations is more important in differentiating schizoaffective disorder from bipolar disorder with psychosis. Not surprisingly, schizoaffective patients continued to have more hallucinatory activity than depression patients over many of the follow-up periods (2, 4.5, 10 and 20 years). Historically, some have viewed schizoaffective patients as primarily mood disorder patients (Pope \& Lipinski, 1978; Pope et al. 1980). Our data, at least with regard to hallucinations, do not confirm this. Schizoaffective patients are more vulnerable to psychotic symptoms (Harrow et al. 2000) and show more frequent hallucinatory activity as compared with bipolar with psychosis patients. In accord with this, during the index phase, schizoaffective patients are similar to schizophrenia patients in hallucinatory status.

Last, bipolar with psychosis patients had more hallucinations at the index hospitalization compared with depression patients, who were included in the sample because they did not have any hallucinations at index. However, as the longitudinal trajectory demonstrated, bipolar patients with psychosis did not differ from unipolar depression patients at any of the follow-ups.

These findings have relevance for our understanding of diagnostic boundaries and differential diagnosis between similar disorders. The results suggest that in differentiating between schizophrenia and schizoaffective disorder, the longer-term course of hallucinations differentiates better than the indexhospitalization phase, whereas, the early years better differentiate schizoaffective disorder from bipolar with psychosis. At least with regards to hallucinatory status, this study also provides support for major aspects of the current diagnostic scheme, with schizophrenia and schizoaffective disorder being grouped together due to a similar early course and bipolar disorder and depression being grouped together. Additionally, schizoaffective disorder shared some commonalities with both schizophrenia and bipolar with psychosis with regards to hallucinatory activity. 


\section{Relationship between hallucinations and real-world functioning}

Across all patients, the presence of a hallucination in the 2-year follow-up period predicted a reduced likelihood of having a future recovery period over the next 18 years. Also, there was a relationship between the presence of hallucinations and lower work attainment at the follow-up periods for all patients. This pattern was also present in schizophrenia patients when analysed separately. These data suggest that hallucinations which persist in the first few years after the acute phase are an indication in many cases of a more severe vulnerability to hallucinations, with this vulnerability influencing subsequent functioning and future outcome. Unfortunately, given that $44 \%$ of schizophrenia patients and $20 \%$ of schizoaffective patients demonstrate frequent or chronic hallucinations, experiencing a period of recovery or achieving adequate work functioning is unlikely for a large group of patients.

These results contrast with previous reports that have suggested that positive symptoms are not a predictor of real-world functioning in schizophrenia (Green, 1996; Green et al. 2000). Green (1996) and Green et al. (2000) indicate that (1) cognitive impairments in schizophrenia are strongly related to poorer functioning and (2) positive symptoms, such as hallucinations, are unrelated to real-world functioning. Our results demonstrate a relationship between hallucinations and real-world functioning. Our data spanning 20 years may differ from single time-point studies, as longitudinal studies may be better able to chronicle the complex relationship between the symptoms and functioning. In support of our finding that hallucinations are related to work attainment and recovery in patients, studies investigating children in the community for hallucinations longitudinally at baseline (Bartels-Velthuis et al. 2010) and 5-year follow-up (Bartels-Velthuis et al. 2011) also demonstrated that hallucinations were related to real-world functioning. Bartels-Velthuis et al. (2011) found a relationship between auditory hallucinations and school functioning at the 5-year follow-up. Children with severe auditory vocal hallucinations had lower school test scores and attended a lower secondary school type than children with mild auditory vocal hallucinations and controls (Bartels-Velthuis et al. 2011). Together these studies underscore the importance of investigating the longitudinal relationship between symptoms and later outcome.

Additionally, cognitive data from the Chicago follow-up study strongly support Green's proposal (Green, 2000) that cognitive impairments are related to poorer work and real-world functioning
(Bonner-Jackson et al. 2010). However, our data also indicate that many other major types of psychopathology, in addition to hallucinations, also lead to impaired real-world functioning, including first-rank symptoms (Rosen et al. 2011), delusions (Racenstein et al. 2002; Harrow et al. 2004; Harrow \& Jobe, 2010), thought disorder (Racenstein et al. 1999) and negative symptoms (Herbener \& Harrow, 2004; Strauss et al. 2010). In this sample, we found that the presence of hallucinations was related to the lack of a period of recovery and poorer work attainment even when controlling for global functioning and delusions.

\section{Relationship between hallucinations and antipsychotic medications}

We found that schizophrenia and schizoaffective patients not on antipsychotic medications had less hallucinatory activity than patients on antipsychotic medications. These findings are consistent with other reports in the literature, which suggest that patients who are no longer in treatment show better global functioning (Johnstone et al. 1990; Harrow \& Jobe, $2007,2010)$ and are more likely than those still on antipsychotic medication to have a period of recovery (Bland et al. 1978; Fenton \& McGlashan, 1987; Harrison et al. 2001; Harrow \& Jobe, 2005, 2007, 2010). These seemingly counterintuitive associations are probably due to the fact that schizophrenia patients not on antipsychotics for prolonged periods are a subgroup of patients with greater resiliency. They have better prognostic factors, better pre-morbid developmental achievements, less vulnerability to anxiety and better cognitive functioning (Harrow et al. 2012). However, it is possible that other factors involving the long-term use of antipsychotics may also have influenced these results (Samaha et al. 2007; Harrow et al. 2012).

\section{Limitations}

We evaluated hallucinations at six follow-up periods over 20 years. A more frequent assessment of hallucinations would be preferential. Second, we had a modest sample of schizoaffective and bipolar with psychosis patients ( 25 patients in each group). A larger sample of schizoaffective and bipolar with psychosis patients would have enabled us to conduct a more thorough analysis of the relationship between hallucinations and recovery and work attainment in those specific groups.

\section{Conclusions}

These results detailing the long-term trajectory of hallucinations in schizophrenia, other psychotic, and mood disorder may be useful in our categorization of 
psychiatric illnesses given the ongoing revisions to our current diagnostic systems. Additionally, as hallucinations are quite frequent in psychosis and one predictor of real-world functioning in all patients, better chronicling of the course of psychotic symptoms could be important in developing specialized treatment plans. Last, there is an increasing literature documenting biological mechanisms that may be involved in hallucinations (Shergill et al. 2000; Hubl et al. 2004; Goghari et al. 2010; Jardri et al. 2011); however, this literature needs to be supplemented with research on the phenomenology and theoretical significance of hallucinations to psychotic and mood disorders.

\section{Acknowledgements}

We thank Bob Faull for his role in data management. Funding was provided by the National Institute of Mental Health (NIMH) (grants no. MH-26341 and no. MH-068688 to M.H.). V.M.G. was funded by a Canadian Institutes of Health Research New Investigator Award and a University of Calgary Start-up Grant.

\section{Declaration of Interest}

None.

\section{References}

Arndt S, Andreasen NC, Flaum M, Miller D, Nopoulos P (1995). A longitudinal study of symptom dimensions in schizophrenia. Prediction and patterns of change. Archives of General Psychiatry 52, 352-360.

Bartels-Velthuis AA, Jenner JA, van de Willige G, van Os J, Wiersma D (2010). Prevalence and correlates of auditory vocal hallucinations in middle childhood. British Journal of Psychiatry 196, 41-46.

Bartels-Velthuis AA, van de Willige G, Jenner JA, van Os J, Wiersma D (2011). Course of auditory vocal hallucinations in childhood: 5-year follow-up study. British Journal of Psychiatry 199, 296-302.

Bland RC, Parker JH, Orn H (1978). Prognosis in schizophrenia. Prognostic predictors and outcome. Archives of General Psychiatry 35, 72-77.

Bleuler E (1950). Dementia Praecox or The Group of Schizophrenias. International Universities Press: New York.

Bonner-Jackson A, Grossman LS, Harrow M, Rosen C (2010). Neurocognition in schizophrenia: a 20-year multifollow-up of the course of processing speed and stored knowledge. Comprehensive Psychiatry 51, 471-479.

Endicott J, Spitzer RL (1978). A diagnostic interview: the Schedule for Affective Disorders and Schizophrenia. Archives of General Psychiatry 35, 837-844.

Endicott J, Spitzer RL, Fleiss JL, Cohen J (1976). The Global Assessment Scale: a procedure for measuring overall severity of psychiatric disturbance. Archives of General Psychiatry 33, 766-771.

Fenton WS, McGlashan TH (1987). Sustained remission in drug-free schizophrenic patients. American Journal of Psychiatry 144, 1306-1309.

Goeree R, Farahati F, Burke N, Blackhouse G, O'Reilly D, Pyne J, Tarride JE (2005). The economic burden of schizophrenia in Canada in 2004. Current Medical Research and Opinion 21, 2017-2028.

Goghari VM, Sponheim SR, Macdonald 3rd AW (2010). The functional neuroanatomy of symptom dimensions in schizophrenia: a qualitative and quantitative review of a persistent question. Neuroscience and Biobehavioral Reviews 34, 468-486.

Green MF (1996). What are the functional consequences of neurocognitive deficits in schizophrenia? American Journal of Psychiatry 153, 321-330.

Green MF, Kern RS, Braff DL, Mintz J (2000). Neurocognitive deficits and functional outcome in schizophrenia : are we measuring the 'right stuff'? Schizophrenia Bulletin 26, 119-136.

Grinker R, Harrow M (1987). Clinical Research in Schizophrenia: A Multidimensional Approach. Thomas CC: Springfield, IL.

Gur RE, Petty RG, Turetsky BI, Gur RC (1996). Schizophrenia throughout life: sex differences in severity and profile of symptoms. Schizophrenia Research 21, 1-12.

Harding CM, Brooks GW, Ashikaga T, Strauss JS, Breier A (1987). The Vermont Longitudinal Study of Persons with Severe Mental Illness, II: long-term outcome of subjects who retrospectively met DSM-III criteria for schizophrenia. American Journal of Psychiatry 144, 727-735.

Harrison G, Hopper K, Craig T, Laska E, Siegel C, Wanderling J, Dube KC, Ganev K, Giel R, An der Heiden W, Holmberg SK, Janca A, Lee PWH, Leon CA, Malhotra S, Marsella AJ, Nakane Y, Sartorius N, Shen Y, Skoda C, Thara R, Tsirkin SJ, Varma VK, Walsh D, Wiersma D (2001). Recovery from psychotic illness: a 15- and 25-year international follow-up study. British Journal of Psychiatry 178, 506-517.

Harrow M, Grossman LS, Herbener ES, Davies EW (2000). Ten-year outcome: patients with schizoaffective disorders, schizophrenia, affective disorders and mood-incongruent psychotic symptoms. British Journal of Psychiatry 177, 421-426.

Harrow M, Herbener ES, Shanklin A, Jobe TH, Rattenbury F, Kaplan KJ (2004). Followup of psychotic outpatients : dimensions of delusions and work functioning in schizophrenia. Schizophrenia Bulletin 30, 147-161.

Harrow M, Jobe TH (2005). Longitudinal studies of outcome and recovery in schizophrenia and early intervention: can they make a difference? Canadian Journal of Psychiatry 50, 879-880.

Harrow M, Jobe TH (2007). Factors involved in outcome and recovery in schizophrenia patients not on antipsychotic medications: a 15-year multifollow-up study. Journal of Nervous and Mental Disease 195, 406-414.

Harrow M, Jobe TH (2010). How frequent is chronic multiyear delusional activity and recovery in 
schizophrenia: a 20-year multi-follow-up. Schizophrenia Bulletin 36, 192-204.

Harrow M, Jobe TH, Faull RN (2012). Do all schizophrenia patients need antipsychotic treatment continuously throughout their lifetime? A 20-year longitudinal study. Psychological Medicine. Published online 17 February 2012. doi:10.1017/S0033291712000220.

Herbener ES, Harrow M (2004). Are negative symptoms associated with functioning deficits in both schizophrenia and nonschizophrenia patients? A 10-year longitudinal analysis. Schizophrenia Bulletin 30, 813-825.

Hubl D, Koenig T, Strik W, Federspiel A, Kreis R, Boesch C, Maier SE, Schroth G, Lovblad K, Dierks T (2004). Pathways that make voices - white matter changes in auditory hallucinations. Archives of General Psychiatry 61, 658-668.

Jardri R, Pouchet A, Pins D, Thomas P (2011). Cortical activations during auditory verbal hallucinations in schizophrenia: a coordinate-based meta-analysis. American Journal of Psychiatry 168, 73-81.

Johnstone EC, Macmillan JF, Frith CD, Benn DK, Crow TJ (1990). Further investigation of the predictors of outcome following first schizophrenic episodes. British Journal of Psychiatry 157, 182-189.

Kraepelin E (1907). Clinical Psychiatry: A Textbook for Students and Physicians. Macmillan Company: London.

Mancevski B, Keilp J, Kurzon M, Berman RM, Ortakov V, Harkavy-Friedman J, Rosoklija G, Dwork AJ (2007). Lifelong course of positive and negative symptoms in chronically institutionalized patients with schizophrenia. Psychopathology 40, 83-92.

Marengo J, Harrow M, Herbener ES, Sands J (2000). A prospective longitudinal 10-year study of schizophrenia's three major factors and depression. Psychiatry Research 97, 61-77.

McGlashan TH (1988). A selective review of recent NorthAmerican long-term follow-up studies of schizophrenia. Schizophrenia Bulletin 14, 515-542.

Pfohl B, Winokur G (1982). The evolution of symptoms in institutionalized hebephrenic catatonic schizophrenics. British Journal of Psychiatry 141, 567-572.

Pope Jr. HG, Lipinski JF, Cohen BM, Axelrod DT (1980). 'Schizoaffective disorder': an invalid diagnosis? A comparison of schizoaffective disorder, schizophrenia, and affective disorder. American Journal of Psychiatry 137, 921-927.

Pope Jr. HG, Lipinski Jr. JF (1978). Diagnosis in schizophrenia and manic-depressive illness : a reassessment of the specificity of 'schizophrenic' symptoms in the light of current research. Archives of General Psychiatry 35, 811-828.

Racenstein JM, Harrow M, Reed R, Martin E, Herbener E, Penn DL (2002). The relationship between positive symptoms and instrumental work functioning in schizophrenia: a 10 year follow-up study. Schizophrenia Research 56, 95-103.

Racenstein JM, Penn D, Harrow M, Schleser R (1999). Thought disorder and psychosocial functioning in schizophrenia: the concurrent and predictive relationships. Journal of Nervous and Mental Disease 187, 281-289.

Rosen C, Grossman LS, Harrow M, Bonner-Jackson A, Faull R (2011). Diagnostic and prognostic significance of Schneiderian first-rank symptoms : a 20-year longitudinal study of schizophrenia and bipolar disorder. Comprehensive Psychiatry 52, 126-131.

Samaha AN, Seeman P, Stewart J, Rajabi H, Kapur S (2007). 'Breakthrough' dopamine supersensitivity during ongoing antipsychotic treatment leads to treatment failure over time. Journal of Neuroscience 27, 2979-2986.

Schultz SK, Miller DD, Oliver SE, Arndt S, Flaum M, Andreasen NC (1997). The life course of schizophrenia: age and symptom dimensions. Schizophrenia Research 23, 15-23.

Shergill SS, Brammer MJ, Williams SC, Murray RM, McGuire PK (2000). Mapping auditory hallucinations in schizophrenia using functional magnetic resonance imaging. Archives of General Psychiatry 57, 1033-1038.

Spitzer RL, Endicott J, Robins E (1978). Research diagnostic criteria: rationale and reliability. Archives of General Psychiatry 35, 773-782.

Strauss GP, Harrow M, Grossman LS, Rosen C (2010). Periods of recovery in deficit syndrome schizophrenia: a 20-year multi-follow-up longitudinal study. Schizophrenia Bulletin 36, 788-799.

Strauss JS, Carpenter Jr. WT (1972). The prediction of outcome in schizophrenia. I. Characteristics of outcome. Archives of General Psychiatry 27, 739-746.

Ventura J, Subotnik KL, Guzik LH, Hellemann GS, Gitlin MJ, Wood RC, Nuechterlein KH (2011).

Remission and recovery during the first outpatient year of the early course of schizophrenia. Schizophrenia Research 132, 18-23.

Waters F, Aleman A, Fernyhough C, Allen P (2012). Report on the inaugural meeting of the International Consortium on Hallucination Research: a clinical and research update and 16 consensus-set goals for future research. Schizophrenia Bulletin 38, 258-262. 